\title{
Stable transformation of a Mamestra brassicae (lepidoptera) cell line with the lepidopteran-derived transposon piggyBac
}

\author{
Mauro Mandrioli ${ }^{\mathrm{a}, *}$, Ernst A. Wimmer ${ }^{\mathrm{b}}$ \\ a Dipartimento di Biologia Animale, Università di Modena e Reggio Emilia, Via Campi 213/D, 41100 Modena, Italy \\ ${ }^{\mathrm{b}}$ Lehrstuhl für Genetik, Universität Bayreuth, Universitätsstrasse 30, NW1, D-95447 Bayreuth, Germany
}

Received 6 September 2002; accepted 28 September 2002

\begin{abstract}
Cabbage moth cells were transfected with the vector pBac\{3xP3-EGFPafm $\}$ and helper phsp-pBac. Seventeen percent of the transfected cells showed stable EGFP-expression. This indicates successful and stable transformation of $M$. brassicae cells with a piggyBac-derived vector. Genomic integration of Bac $\{3 x P 3-E G F P a f m\}$ in stably transformed cells was confirmed by Southern blots and inverse PCR. Since the integrations are stable, and transfection with pBac\{3xP3-EGFPafm $\}$ alone did not yield in transformations, no cross-reacting transposase activity seems present in $M$. brassicae cells. Moreover, Southern blotting with a probe for piggyBac transposase indicated the absence of piggyBac-related elements in the genome of Mamestra brassicae. Due to the tissue specificity of the 3xP3-EGFP marker for eye and nervous tissues, it is intriguing that 3xP3-EGFP can successfully be used to identify stably transformed $M$. brassicae cells of cell line IZD-MB0503, which is hemocyte-derived. Sequence analysis of the insertion sites showed that piggyBac inverted repeats were adjacent to TTAA sequences on both termini in all the clones. The present results are particularly important as they suggest that piggyBac can be used for transgenesis of cabbage moth cells.
\end{abstract}

(C) 2002 Elsevier Science Ltd. All rights reserved.

Keywords: Mamestra brassicae; Cabbage moth; piggyBac; Genetic transformation

\section{Introduction}

Mamestra brassicae is a cosmopolitan and polyphagous insect that lives in temperate regions of Eurasia on cruciferae plants. M. brassicae control in the field is performed using both chemical compounds and Bacillus thuringensis (Bt) toxins (Kwa et al., 1998). The low specificity of the used insecticides together with the diffusion of resistance to $\mathrm{Bt}$ endotoxins in Lepidoptera (Tabashnik et al., 1994) necessitates the design of new control strategies for $M$. brassicae. In view of results obtained with other pest crop insects (reviewed in Handler, 2001; Atkinson et al., 2001), genetic engineering techniques will allow the development of new strategies for $M$. brassicae control in the field.

Moreover, M. brassicae cell lines have a wide range

* Corresponding author: Tel.: +39-059-2055544; fax +39-0592055548.

E-mail addresses: mandriol@unimo.it (M. Mandrioli); ernst.wimmer@uni-bayreuth.de (E.A. Wimmer). of applications for the production of recombinant proteins, for studies on baculoviruses and for the analysis of apoptosis in insect cells (e.g. Wickham et al., 1992). Mutagenesis by insertion of transposable elements into the genome of such a cell line could be particularly interesting to identify and characterize the genes involved in apoptosis of insect cells.

Transposable elements have become an essential tool in the study of gene function in insects and their applications range from the use as mutagens to their use as tags for the identification of new genes. In particular, efforts have focused on the identification and molecular characterization of mobile elements that will serve as efficient gene vectors. Insect transformation is, in fact, an emerging technology that is likely to have a wide range of applications within insect biology and genetics (reviewed in Handler and James, 2000; Handler, 2001; Atkinson et al., 2001).

The successful improvement of this technology allowed the development of transgenic insects that could be useful to develop new control strategies for insects 
of agricultural and medical interest. In this regard, the transposable element piggyBac appeared particularly interesting since it has been used to achieve genetic transformation in several insect species of agricultural, economical and medical interest, such as Ceratitis capitata, Bactrocera dorsalis, Anastrepha suspensa, Musca domestica, Lucilia cuprina, Anopheles gambiae, An. stephensi, An. albimanus, Aedes aegypti, Pectinophora gossypiella, Bombyx mori, and Tribolium castaneum insects (reviewed in Handler, 2002).

The piggyBac element is a short inverted terminal repeat (ITR) transposable element, $2.5 \mathrm{~kb}$ long, with 13 bp ITR sequences and a single $2.1 \mathrm{~kb}$ ORF (Cary et al., 1989; Elick et al., 1996). It was originally discovered as a result of it causing few polyhedra mutations in baculoviruses that had been passed through the TN-368 cell line of cabbage looper moth Trichoplusia ni (Fraser et al., 1995). Subsequently, its ability to integrate in different lepidopteran and Anopheles cell lines (Fraser et al., 1995; Grossman et al., 2000) and in germ cells of numerous insects (reviewed in Handler, 2002) has been shown.

In the present paper, we report that the piggyBac transposable element from the cabbage looper T. $n i$ can be used for the effective and stable transformation of a $M$. brassicae cell line and that the 3xP3-EGFP marker gene (Berghammer et al., 1999; Horn et al., 2000, 2002) can be used to identify such lines. This represents the first successful transformation of a cabbage moth cell line.

\section{Materials and methods}

The IZD-MB-0503 cell line from the insect M. brassicae (Lepidoptera) (ATCC number: CRL-8003) was used. The cells were cultured in Ex-Cell 405 medium (JRH Biosciences, KS, USA) at $26^{\circ} \mathrm{C}$.

Genomic DNA extraction from the IZD-MB-0503 cell line was performed according to Mandrioli (2002). Electrophoresis, transfer of the DNA from agarose gel to nylon membrane and Southern blotting were performed following Mandrioli (2002).

Construction of the $\mathrm{pBac}\{3 \times \mathrm{xP} 3$-EGFPafm $\}$ vector is described in Horn and Wimmer (2000). The helper plasmid, phsp-pBac, contains the piggyBac transposase sequence under the control of the Drosophila hsp 70 promoter (Handler and Harrell, 2001).

Transfection of the cell with both helper phsp-pBac and $\mathrm{pBac}\{3 \mathrm{xP} 3$-EGFPafm $\}$ plasmids were performed using DOTAP liposomes (Roche) following manufacturer protocols. After a $48 \mathrm{~h}$ incubation after transfection, transposase expression was induced by heat-shock $(1 \mathrm{~h}$ at $37^{\circ} \mathrm{C}$ ). Twelve hours after heat-shock, EGFP-fluorescent cells were isolated from non-fluorescent ones using a flow cytometer equipped with a cell sorter.

Primers PLF (5'-CTTGACCTTGCCACAGAGGACT
ATTAGAGG) and PRR (5'-AGTCAGTCAGAAACA ACTTTGGCACATATC) were used to PCR-amplify the EGFP cassette, whereas inverse PCRs was performed according to Hediger et al. (2001). PCR fragments were separated in a $1.2 \%$ TAE agarose gel, purified with the Agarose Gel Extraction kit (Roche), cloned, and sequenced. The amplified fragments were cloned with the 'pGEMT-easy cloning kit' following the Promega protocols. Sequencing was performed at the 'CRIBISequencing Centre of the University of Padua'.

\section{Results and discussion}

When cabbage moth cells had been transfected with both $\mathrm{pBac}\{3 \times \mathrm{xP} 3-\mathrm{EGFPafm}\}$ as well as helper plasmids and transposase expression had been induced by heatshock, $17 \%$ of the transfected cells resulted in stable EGFP expression and could therefore be considered successfully transformed with a piggyBac-derived vector (Fig. 1B). In order to evaluate if piggyBac-integration was due to the presence of the helper plasmid or to a phenomenon of cross-mobilization, the presence of endogenous transposase able to mobilize piggyBac in $M$. brassicae cells was assessed by transfecting cells with
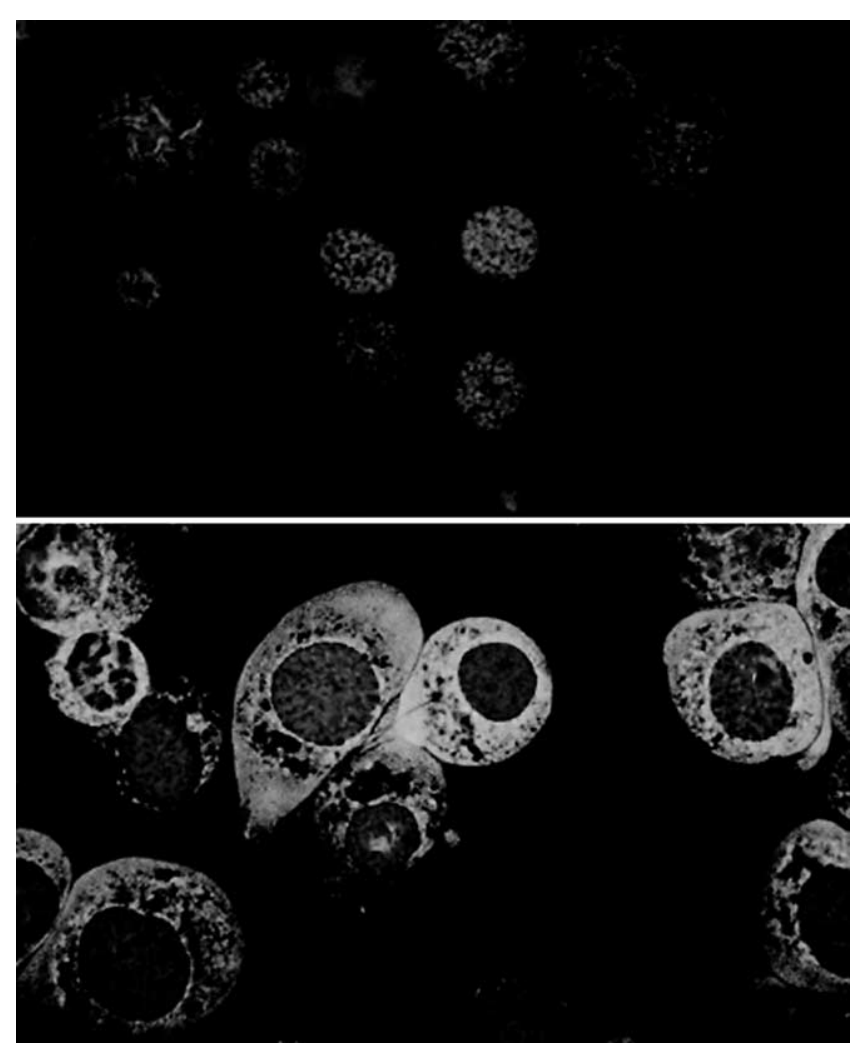

Fig. 1. M. brassicae cells have been transfected with the $\mathrm{pBac}\{3 \mathrm{xP} 3-$ EGFPafm $\}$ plasmid alone (A) or with both helper and pBac\{3xP3EGFPafm \} plasmids (B). Stable EGFP expression was obtained $12 \mathrm{~h}$ after heat-shock using both plasmids only. In both panels the cell nuclei are labeled by the DNA dye DAPI. 
the $\mathrm{pBac}\{3 \mathrm{xP} 3-\mathrm{EGFPafm}\}$ vector alone (Fig. 1A). No stable EGFP-expressing cells were obtained and only transient EGFP expression could be observed. Moreover, the presence of piggyBac-like elements was evaluated in the cabbage moth genome by Southern blots using the DIG-labeled piggyBac transposase gene as a probe. No hybridization signal was observed even when low stringency conditions were tried. This indicates the absence of piggyBac-related elements from the M. brassicae genome.

To verify stable integration of $B a c\{3 x P 3-E G F P a f m\}$ into the genome, genomic DNA was extracted from stable EGFP-expressing and control $M$. brassicae cells not expressing EGFP. This DNA was initially tested by PCR with primers PLF and PRR and by Southern blotting. PCR amplified DNA was found in all the EGFP expressing cell lines, whereas no PCR products were recovered from non-fluorescent cells (Fig. 2, lanes 1 and 2 ). These results strongly suggested that successful transformation of $M$. brassicae cells occurred, since fluorescence of transformed cells was correlated to the presence of the EGFP gene. To further verify these results, $M$. brassicae genomic DNA from transformed and non transformed cells were digested with restriction enzymes

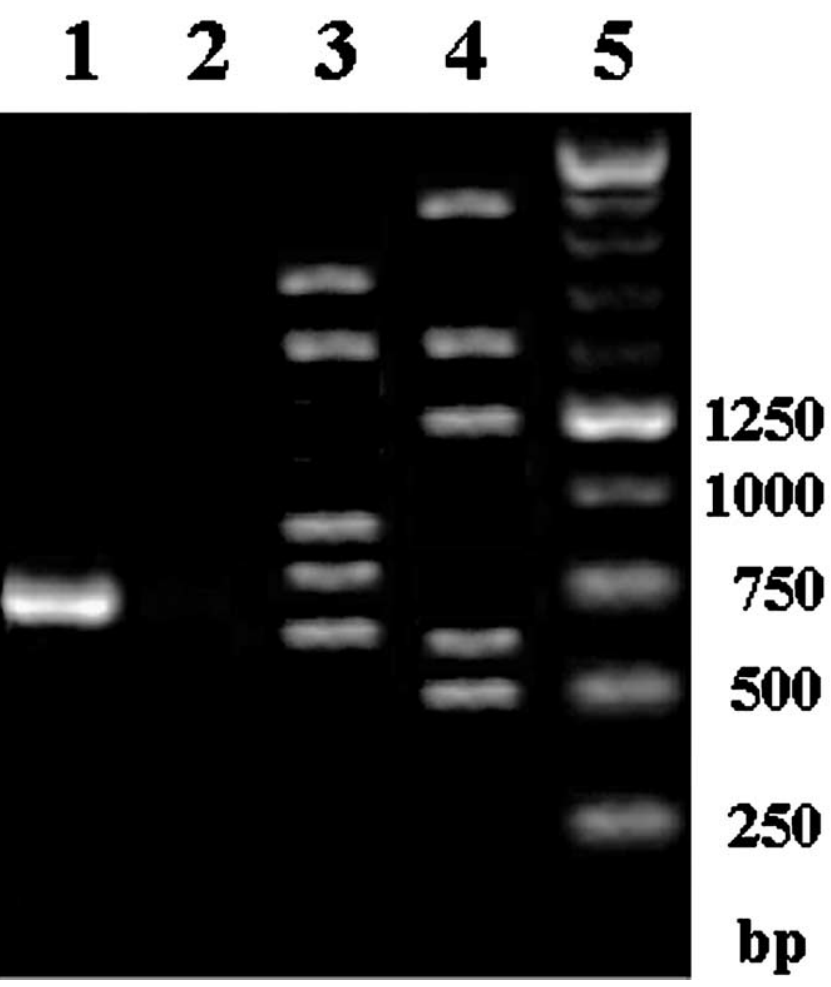

Fig. 2. PCR amplification showed the presence of the EGFP cassette in the EGFP expressing lane (lane 1) whereas no PCR products were recovered from non-fluorescent cells (lane 2). Inverse PCRs, performed using DNA extracted from EGFP-expressing cells, evidenced five bands corresponding to different piggyBac right ends (lane 3) and five bands corresponding to left ends (lane 4). The molecular weight of the amplified fragments has been evaluated using the $500 \mathrm{bp}$ ladder (lane 5). and labelled with the EGFP probe obtained by digesting the pBac\{3xP3-EGFPafm $\}$ plasmid with BamHl and Notl. All the EGFP expressing cell lines showed a 750 bp band after simultaneous digestion with BamHl and Not l corresponding to the EGFP cassette (Fig. 3A, lane 3). Moreover, 2-5 bands were detected in the EGFPexpressing cells when digested with only one of the restriction enzymes (Fig. 3A, lanes 1 and 2). This indicates that the EGFP construct was inserted into several different genomic sites and that piggyBac was indeed an effective vector for $M$. brassicae cell line transformation. No hybridization signal was observed with the EGFP probe on Southern blots of cells not expressing EGFP (Fig. 3B).

In order to identify the piggyBac insertion sequences in the cabbage moth cells, inverse PCR was performed using DNA extracted from EGFP-expressing cells. piggyBac left and right flanking sequences were separately amplified as described (Hediger et al., 2001), and five amplified fragments (Fig. 2, lanes 3 and 4) were cloned and sequenced for each terminal repeat. Analysis of the sequenced clones showed that piggyBac inverted repeats were adjacent to a TTAA sequence on both the termini in all the clones (Table 1). Moreover, sequence analysis performed using alignment with GCG software did not reveal further significant similarities among the insertion sequences amplified by inverse PCR. These results allowed us to conclude that piggyBac insertion into the $M$. brassicae genome was due to a specific transposasemediated mechanism. These data are in full agreement with those reported for An. gambiae, A. suspensa, Spodoptera frugiperda and M. domestica (Elick et al., 1996; Grossman et al., 2000; Handler and Harrell, 2001;

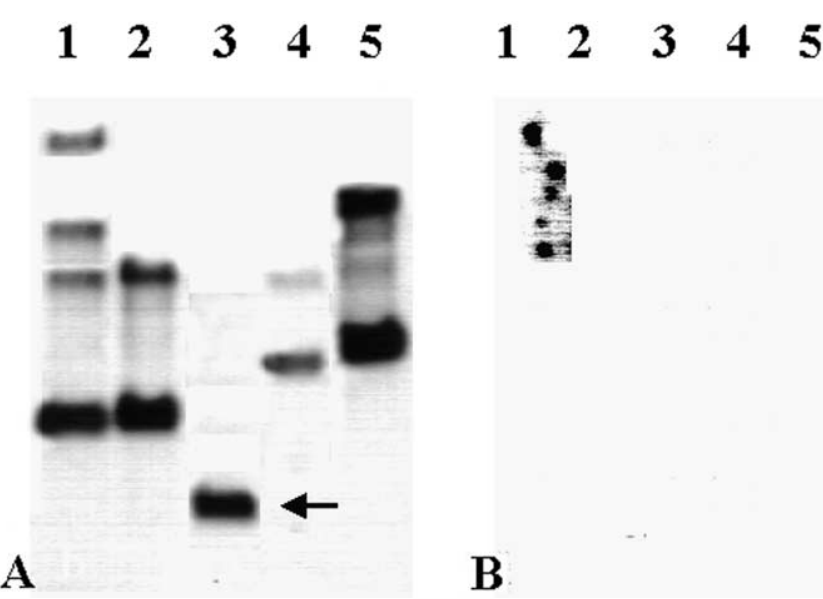

Fig. 3. M. brassicae genomic DNA from transformed (A) and nontransformed cells (B) were digested with BamHI (lane 1), NotI (lane 2, BamHI and Not1 (lane 3), EcoRI (lane 4) and Sau3A (lane 5) and probed with the BamHI-NotI EGFP fragment from pBac\{3xP3EGFPafm $\}$. The EGFP expressing transformed cell lines showed a 750 bp band after simultaneous digestion with BamHI and NotI corresponding to the EGFP cassette. With single restriction enzyme digests more bands were observed, which indicates multiple piggyBac insertions. 
Table 1

Inverse PCR strategy was used to identify the genomic sequences flanking the insertion sites in the transformed cells. Left and right flanking sequences were separately amplified according to Hediger et al. (2001). Comparison of the piggyBac flanking sequences amplified by inverse PCR indicated that in all cases, the TTAA sequence flanked piggyBac. The TTAA sequence is duplicated by transposasemediated integration

\begin{tabular}{ll}
\hline Clone & Left end \\
\hline L1 & tagctcgctagatagactcgtagTTAA - piggyBac \\
L2 & gtagctacgctcgatagctaccTTAA - piggyBac \\
L3 & atttagcgctagctcgatcgcttTTAA - piggyBac \\
L4 & gagctgggcatagactcccaTTAA - piggyBac \\
L5 & cgecccttacagacatagcaTTAA - piggyBac \\
\hline & Right end \\
R1 & piggyBac - TTAActgctagctcgatagatttt \\
R2 & piggyBac - TTAAtagctagctttagatcttgt \\
R3 & piggyBac - TTAAtagcttagctcgctcgctgtgg \\
R4 & piggyBac - TTAAgggtgatagatagatgctcgc \\
R5 & piggyBac - TTAAcgaccttcatcgctcgttaa \\
\hline
\end{tabular}

Hediger et al., 2001) and strongly reinforce the hypothesis that the TTAA sequence is the integration consensus sequence of piggyBac. The structure of piggyBac integration sequence has been contradictory since Cary et al. (1989) suggested the existence of a larger piggyBac consensus sequence consisting of YYTTTTTT/AARTAAYAG $(\mathrm{Y}=$ pyrimidine, $\quad \mathrm{R}=$ purine, / = insertion sequence). The same hypothesis has been proposed in An. gambiae (Grossman et al., 2000). Our results do not support this but reinforce the existence of a short TTAA piggyBac insertion sequence.

The present results indicate that the marker 3xP3EGFP can be successfully used to identify stably transformed M. brassicae cells of line IZD-MB-0503. This is particularly intriguing as this cell line is supposed to be of hemocyte origin (Miltenburger and David, 1976), but the 3xP3-EGFP marker is supposedly tissue specific for eye and nervous system tissues (Horn et al., 2000). It is possible, that the 3xP3-EGFP marker itself does not express EGFP stably in this cell line. This would explain why plasmid-based marker gene expression is only very transiently detected. However, after insertion of the marker gene into the genome, enhancer trap effects might allow for strong expression of EGFP. Enhancers close enough to the $3 \times \mathrm{xP}$-EGFP insertion might activate the basal promoter of $3 \times \mathrm{xP}$ (Horn et al., 2000) and thus mediate stable EGFP expression.

In any case, the availability of a highly efficient transformation vector based on piggyBac will be particularly interesting for the study of gene expression in the $M$. brassicae IZD-MB-0503 cell line. This will allow for the quick evaluation of promoter strengths in a genomic environment. Expression libraries constructed in piggyBac vectors could be functionally screened in the $M$. brassicae cell line. In particular, it will be interesting to integrate gene constructs that could be used to perform RNA-interference experiments. Furthermore the stable integration suggests that piggyBac could also be employed for transgenesis of cabbage moth eggs and embryos.

\section{Acknowledgements}

This work is supported by 'Fondi MURST riservati a giovani ricercatori per il finanziamento di attività di Rierca' from Ministero della Ricerca Scientifica e Tecnologica (M.U.R.S.T.) of Italy (M.M.) and the Robert Bosch Foundation (E.A.W.). In addition, E.A.W. receives support as an EMBO Young Investigator.

\section{References}

Atkinson, P.W., Pinkerton, A.C., O’Brochta, D.A., 2001. Genetic transformation systems in insects. Ann. Rev. Entomol. 46, 317346.

Berghammer, A.J., Klingler, M., Wimmer, E.A., 1999. A universal marker for transgenic insects. Nature 402, 370-371.

Cary, L.C., Goebel, M., Corsaro, B.G., Wang, H.G., Rosen, E., Fraser, M.J., 1989. Transposon mutagenesis of baculoviruses: analysis of Trichoplusia ni transposon IFP2 insertions within the FP-locus of nuclear polyhedrosis viruses. Virology 172, 156-169.

Elick, T.A., Bauser, C.A., Fraser, M.J., 1996. Excision of the piggyBac transposable element in vitro is a precise event that is enhanced by the expression of its encoded transposase. Genetica 98, 33-41.

Fraser, M.J., Cary, L., Boonvisudhi, K., Wang, H.G.H., 1995. Assay for movement of lepidopteran transposon IFP2 in insect cells using $\alpha$ baculovirus genome as target DNA. Virology 211, 397-407.

Grossman, G.L., Rafferty, C.S., Fraser, M.J., Benedict, M.Q., 2000. The piggyBac element is capable of precise excision and transposition in cell and embryos of the mosquito Anopheles gambiae. Insect Biochem. Mol. Biol. 30, 909-914.

Handler, A.M., James, A.A., 2000. Insect Transgenesis: Methods and Applications. CRC Press, Boca Raton, FL.

Handler, A.M., Harrell, R.A., 2001. Transformation of the Caribbean fruit fly, Anastrepha suspensa, with a piggyBac vector marked with polyubiquitin-regulated GFP. Insect Biochem. Mol. Biol. 31, 199-205.

Handler, A.M., 2001. A current perspective on insect gene transformation. Insect Biochem. Mol. Biol. 31, 11-28.

Handler, A.M., 2002. Use of the piggyBac transposon for germ line transformation of insects. Insect Biochem. Mol. Biol 32, 12111220.

Hediger, M., Niessen, M., Wimmer, E.A., Dubendorfer, A., Bopp, D., 2001. Genetic transformation of the housefly Musca domestica with the lepidopteran derived transposon piggyBac. Insect. Biochem. Mol. Biol. 10, 113-119.

Horn, C., Jaunich, B., Wimmer, E.A., 2000. Highly sensitive, fluorescent transformation marker for Drosophila transgenesis. Dev. Genes Evol. 210, 623-629.

Horn, C., Wimmer, E.A., 2000. A versatile vector set for animal transgenesis. Dev. Genes Evol. 210, 630-637.

Horn, C., Schmid, B.G.M., Pogoda, F.S., Wimmer, E.A., 2002. Fluorescent transformation markers for insect transgenesis. Insect Biochem. Mol. Biol. 32, 1221-1235.

Kwa, M.S., de Maagd, R.A., Stiekema, W.J., Vlak, J.M., Bosch, D., 
1998. Toxicity and binding properties of the Bacillus thuringiensis delta-endotoxin Cry1C to cultured insect cells. J. Invert. Pathol. 71, 121-127.

Mandrioli, M., 2002. Cytogenetic characterization of telomeres in the holocentric chromosomes of the lepidopteran Mamestra brassicae. Chromosome Res. 9, 279-286.

Miltenburger, H.G., David, P., 1976. Nuclear polyhedrosis virus replication in permanent cell lines of the cabbage moth (Mamestra brassicae L.). Naturwissenschaften 63, 197-198.
Tabashnik, B.E., Finson, N., Groeters, F.R., Moar, W.J., Johnson, M.W., Luo, K., Adang, M.J., 1994. Reversal of resistance to Bacillus thuringiensis in Plutella xylostella. Proc. Natl Acad. Sci. USA 91, 4120-4124.

Wickham, T.J., Davis, T., Granados, R.R., Shuler, M.I., Wood, H.A., 1992. Screening of insect cell lines for the production of recombinant proteins and infectious virus in the baculovirus expression systems. Biotechnol. Prog. 8, 391-396. 